Proyecciones

Vol. 23, No 1, pp. 31-49, May 2004.

Universidad Católica del Norte

Antofagasta - Chile

\title{
GALERKIN APPROXIMATION FOR A SEMI LINEAR PARABOLIC PROBLEM WITH NONLOCAL BOUNDARY CONDITIONS
}

\author{
ABDESSLAM BOUTAYEB \\ ABDELAZIZ CHETOUANI \\ Université Mohammed Premier, Maroc \\ Received March 2003. Accepted November 2003.
}

\begin{abstract}
We analyze a $\theta$-method for the numerical solution of a semi linear parabolic problem with boundary conditions containing integrals over the interior of the interval. Existence and convergence are proved for $\theta \geq 1 / 2$, numerical application is given.
\end{abstract}

Keywords : Semi linear parabolic problem, nonlocal boundary conditions, $\theta$-Galerkin method.

AMS classification : 65M60, 65M15, 35K55. 


\section{Introduction}

This paper is concerned with the numerical solution of the semi linear parabolic problem

$$
\left\{\begin{array}{l}
u_{t}-\left(a(x) u_{x}\right)_{x}-b(x) u_{x}=F(u, x, t) \\
\alpha<x<\beta, \quad 0<t \leq T
\end{array}\right.
$$

subject to the nonlocal boundary conditions

$$
\left\{\begin{array}{l}
u(\alpha, t)=\int_{\alpha}^{\beta} K_{0}(x) u(x, t) d x+g_{0}(t) \\
u(\beta, t)=\int_{\alpha}^{\beta} K_{1}(x) u(x, t) d x+g_{1}(t) \\
0 \leq t \leq T
\end{array}\right.
$$

and the initial condition

$$
u(x, 0)=u_{0}(x), \quad \alpha \leq x \leq \beta .
$$

We assume that the function $F$ is Lipschitz continuous on compact sets of $\mathbf{R}$, the functions $a$ and $b_{x}$ are supposed to be continuous and that there exist positive constants $a_{1}, a_{2}, b_{1}, b_{2}$ such that

$$
a_{1} \leq a(x) \leq a_{2} \text { and } b_{1} \leq b_{x}(x) \leq b_{2}, \quad \alpha \leq x \leq \beta .
$$

This kind of initial-boundary-value problem arises in the quasi-static theory of thermoelasticity $[2,3]$. The existence, uniqueness and some analytic properties of the solution of (1.1)-(1.3), have been studied by Day [2, 3], Friedman [6], Kawohl [7], when $b(x)=0, \alpha=0$ and $\beta=1$ under the hypothesis that

$$
\int_{0}^{1}\left|K_{0}(x)\right| d x<1, \quad \int_{0}^{1}\left|K_{1}(x)\right| d x<1 .
$$

We assume that the functions $u, u_{0}, g_{0}, g_{1}$ are sufficiently smooth and the functions $K_{0}$ and $K_{1}$ satisfy

$$
\begin{aligned}
& (\beta-\alpha) \int_{\alpha}^{\beta}\left|K_{0}(x)\right|^{2} d x \leq \mu^{2}<1, \\
& (\beta-\alpha) \int_{\alpha}^{\beta}\left|K_{1}(x)\right|^{2} d x \leq \mu^{2}<1 .
\end{aligned}
$$

Fairweather and López-Marcos [5] considered the Crank-Nicholson method and the extrapolated Crank-Nicholson method for the problem (1.1)-(1.3) 
when $b(x)=0, \alpha=0, \beta=1$ using an energy argument, they proved the convergence of both methods under the condition (1.6), Fairweather, López-Marcos and A.Boutayeb [11] analyzed an orthogonal spline collocation method for a quasi linear parabolic problem, recently the present authors [1] considered several methods of extrapolations for a linear parabolic problem with non local boundary conditions.

The main purpose of this paper is to show the convergence of a $\theta$-finite element Galerkin method ( $\theta$-method) for the solution of (1.1-1.3), and for $\theta \in\left[\frac{1}{2}, 1\right]$, using similar energy argument to the one in [5]. This paper is outlined as follows: Section 2 is devoted to the $\theta$ - method while the consistency, the stability, the convergence and the existence of the $\theta$-method are treated in section 3,4 and 5. In the last section we give some numerical results.

\section{The $\theta$-method}

According to [5], let be $\left(\pi_{h}\right)=\left(x_{j}\right)_{j=0}^{J}$ a partition of $[\alpha, \beta]$ such that $\alpha=x_{0}<x_{1}<\ldots<x_{J}=\beta$, where $h=\max _{j}\left(h_{j}\right), h_{j}=x_{j}-x_{j-1}$ and $I_{j}=\left[x_{j-1}, x_{j}\right], 1 \leq j \leq J$, we consider a family of partitions $\left(\pi_{h}\right)_{h \in H}$ where $H$ is a set of positif numbers with $\inf (H)=0$, we define the space $S_{h}$ in the following way

$$
S_{h}=\left\{v / v \in C^{n}[\alpha, \beta] \text { and } v / I_{j} \in \mathbb{P}_{r}\left(I_{j}\right), 1 \leq j \leq J\right\},
$$

and $S_{h}^{\circ}$, the subspace of $S_{h}$, given by

$$
S_{h}^{\circ}=\left\{v \in S_{h} / V(\alpha)=V(\beta)=0\right\} .
$$

Where $\mathbb{P}_{r}(E)$ denotes the set of polynomials of degree at most $r, r \geq 2$ for a closed interval $E$ and $n<r$.

We denote by $k$ the time step, $t_{m}=m k, m=0, \ldots, M=[T / k], t_{m+\theta}=$ $(m+\theta) k$, and $(.,$.$) the usual inner product on L^{2}([\alpha, \beta])$.

The $\theta$-Galerkin approximation $\left(\theta\right.$ - method), $\left(U^{m}\right)_{m=0}^{M} \subset S_{h}$ to the solution of (1.1-1.3) is defined by

$$
\begin{array}{r}
\left(\frac{U^{m+1}-U^{m}}{k}, v\right)+\left(a U_{x}^{m+\theta}, v_{x}\right)-\left(b U_{x}^{m+\theta}, v\right)-\left(F\left(U^{m+\theta}, t_{m+\theta}\right), v\right)=0 \\
v \in S_{h}^{0},
\end{array}
$$

$0 \leq m \leq M-1$, where $U^{m+\theta}=\theta U^{m+1}+(1-\theta) U^{m}$. 
With the nonlocal boundary conditions

$$
\begin{aligned}
& U^{m+1}(\alpha)=\int_{\alpha}^{\beta} K_{0}(x) U^{m+1}(x) d x+g_{0}\left(t_{m+1}\right), \\
& U^{m+1}(\beta)=\int_{\alpha}^{\beta} K_{1}(x) U^{m+1}(x) d x+g_{1}\left(t_{m+1}\right),
\end{aligned}
$$

$0 \leq m \leq M-1, U^{0} \in S_{h}$ will be specified later.

For the analysis of this method we require to examine the consistency (sect.3) and the stability (sect.4) of the method, in order to prove the existence and convergence, according to the framework developed in $[8,10]$. In the sequel $C$ denotes a positive constant whose value is not the same on each occurrence and which is independent of $h$ and $k$, we define the norms $\|\cdot\|$ and $\|\cdot\|_{\infty}$, by

$$
\|v\|=\sqrt{(v, v)}, \quad\|v\|_{\infty}=\max _{\alpha \leq x \leq \beta}|v(x)| .
$$

Let $p_{h}: H_{0}^{1}([\alpha, \beta]) \longrightarrow S_{h}^{0}$ be the operator defined by

$$
\left(a\left(z-p_{h}(z)\right)_{x}, v_{x}\right)-\left(b\left(z-p_{h}(z)\right)_{x}, v\right)=0, \quad v \in S_{h}^{0}
$$

we note that

$$
\left\|z-p_{h}(z)\right\|_{\infty} \leq C\|z\|_{W^{r, \infty}(0,1)}, \quad z \in H_{0}^{1}([\alpha, \beta]),
$$

where $H_{0}^{1}([\alpha, \beta])$ is the usual Sobolev space on $[\alpha, \beta],\|v\|_{W^{r, \infty}(0,1)}=\sum_{i=0}^{r}\left\|v^{(i)}\right\|_{\infty}$ ( see for instance Wheeler [9]).

We define on $S_{h}^{0}$ the linear form

$$
\begin{aligned}
& \Gamma^{m+\theta}(v, w)(\chi)=\left(\frac{v-w}{k}, \chi\right)+\left(a\left(\theta v_{x}+(1-\theta) w_{x}\right), \chi_{x}\right) \\
& -\left(b\left(\theta v_{x}+(1-\theta) w_{x}\right), \chi\right)-\left(F\left(\theta v+(1-\theta) w, t_{m+\theta}\right), \chi\right)
\end{aligned}
$$

and the operators $L_{0}$ and $L_{1}$

$$
L_{0}(V)=V(\alpha)-\int_{\alpha}^{\beta} K_{0}(x) V(x) d x, \quad L_{1}(V)=V(\beta)-\int_{\alpha}^{\beta} K_{1}(x) V(x) d x .
$$

We denote

$$
X_{h k}=\left(S_{h}\right)^{M+1} \text { and } Y_{h k}=S_{h} \times\left(\left(S_{h}^{0}\right)^{*}\right)^{M} \times \mathbf{R}^{M} \times \mathbf{R}^{M}
$$


where $\left(S_{h}^{0}\right)^{*}$ is the dual space of $S_{h}^{0}$. It is clear that

$$
\operatorname{dim} X_{h k}=\operatorname{dim} Y_{h k}
$$

We also define the operator

$$
\Phi_{h k}: X_{h k} \longrightarrow Y_{h k}
$$

by the relations

$$
\begin{gathered}
\Phi_{h k}\left(V^{0}, \ldots, V^{M}\right)=\left(U^{0}-V^{0},\left(\Gamma^{m-1+\theta}\left(V^{m}, V^{m-1}\right)\right)_{m=1}^{M}\right. \\
\left(L_{0}\left(\frac{V^{m}-V^{m-1}}{k}\right)-\frac{g_{0}\left(t_{m}\right)-g_{0}\left(t_{m-1}\right)}{k}\right)_{m=1}^{M}, \\
\left.\left(L_{1}\left(\frac{V^{m}-V^{m-1}}{k}\right)-\frac{g_{1}\left(t_{m}\right)-g_{1}\left(t_{m-1}\right.}{k}\right)_{m=1}^{M}\right)
\end{gathered}
$$

given an initial condition $U^{0} \in S_{h}$ that satisfies

$$
L_{0}\left(U^{0}\right)=g_{0}(\alpha), \quad L_{1}\left(U^{0}\right)=g_{1}(\alpha)
$$

It is easy to show that an element $\left(U^{0}, \ldots, U^{M}\right) \in\left(S_{h}\right)^{M+1}$ is a solution of the $\theta$-method if and only if

$$
\Phi_{h k}\left(U^{0}, \ldots, U^{M}\right)=0,
$$

we also need in the analysis the norm

$$
\|\Gamma\|_{*}=\sup _{\chi \in S_{h}^{\circ}} \frac{|\Gamma(\chi)|}{\|\chi\|} \quad \chi \neq 0 .
$$

It is well known that if $K_{0}$ and $K_{1}$ are zero then the $\theta$-method is convergent for $\theta \geq \frac{1}{2}$ and the order of convergence is $O\left(h^{r}+k^{l}\right)$, where $l=1$ if $\theta>\frac{1}{2}$, and $l=2$ if $\theta=\frac{1}{2}$, our aim is to show that a similar result holds in the case of nonlocal boundary conditions. 


\section{Consistency}

We consider the problem

$$
\left\{\begin{array}{l}
\left(a(x)\left(v_{i}\right)_{x}\right)_{x}-b(x)\left(v_{i}\right)_{x}=0, \quad i=0,1 \\
v_{0}(\alpha)=1, v_{0}(\beta)=0, v_{1}(\alpha)=0, v_{1}(\beta)=1
\end{array}\right.
$$

Whose solutions are the positive functions

$$
v_{0}(x)=1-v_{1}(x), \quad v_{1}(x)=\frac{\phi(x)}{\phi(\beta)}
$$

where

$$
\phi(x)=\int_{\alpha}^{x} \frac{1}{a(u)}\left[\exp \left(\int_{\alpha}^{u} \frac{b(t)}{a(t)} d t\right)\right] d u,
$$

and the functions $\phi_{0}, \phi_{1} \in S_{h}$, defined by

$$
\left\{\begin{array}{l}
\left(a(x)\left(\phi_{i}\right)_{x}, \chi_{x}\right)-\left(b(x)\left(\phi_{i}\right)_{x}, \chi\right)=0, \quad i=0,1, \quad \chi \in S_{h}^{0} \\
\phi_{0}(\alpha)=1, \phi_{0}(\beta)=0, \phi_{1}(\alpha)=0, \phi_{1}(\beta)=1
\end{array}\right.
$$

Clearly $\phi_{0}$ and $\phi_{1}$ are the elliptic projections of the functions $v_{0}$ and $v_{1}$, respectively, and from (2.4) we have

$$
\left\|\phi_{i}-v_{i}\right\|_{\infty} \leq C h^{r}
$$

it is easy to show that

$$
\begin{gathered}
\left(v_{0}, v_{1}\right)=\frac{1-\left\|v_{0}\right\|^{2}-\left\|v_{1}\right\|^{2}}{2}, \\
\left\|A v_{0}+B v_{1}\right\|_{\infty}=\max (|A|,|B|),
\end{gathered}
$$

for arbirary constants $A, B \in \mathbf{R}$.

Furthermore, for $\nu>0$ there exists $h_{0}>0$ such that for each $h \leq h_{0}$,

$$
\left(\left|\phi_{0}\right|,\left|\phi_{1}\right|\right) \leq \frac{1-\left\|\phi_{0}\right\|^{2}-\left\|\phi_{1}\right\|^{2}+\nu}{2},
$$

and

$$
\left\|A \phi_{0}+B \phi_{1}\right\|_{\infty} \leq(1+\nu) \max (|A|,|B|) .
$$

For $u(x, t)$ the solution of the problem (1.1-1.3) we define $\hat{u}(x, t) \in H_{1}^{0}([\alpha, \beta])$ by

$$
\hat{u}(x, t)=u(x, t)-u(\alpha, t) v_{0}(x)-u(\beta, t) v_{1}(x),
$$


and $\tilde{u}_{h}(x, t) \in S_{h}$ by

$$
\tilde{u}_{h}(x, t)=P_{h}(\hat{u}(x, t))-u(\alpha, t) \phi_{0}(x)-u(\beta, t) \phi_{1}(x),
$$

we note that

$$
p_{h}\left(\hat{u}_{t}(t)\right)=\left(p_{h}(\hat{u}(t))\right)_{t} .
$$

We set

$$
\tilde{u}_{h}^{m}=\tilde{u}_{h}\left(t_{m}\right), \quad 0 \leq m \leq M .
$$

We have then the following theorem

Theorem 3.1. Suppose that the solution of the problem (1.1-1.3) is in $C^{r, 3}([\alpha, \beta] \times[0, T])$. For $h$ sufficiently small and $0 \leq m \leq M-1$,

$$
\left\|\Gamma^{m+\theta}\left(\tilde{u}_{h}^{m+1}, \tilde{u}_{h}^{m}\right)\right\|_{*}=\left\{\begin{array}{l}
O\left(h^{r}+k^{2}\right), \quad \theta=1 / 2, \\
O\left(h^{r}+k\right), \quad \theta>1 / 2
\end{array}\right.
$$

and for $i=0,1$

$$
\left|L_{i}\left(\frac{\tilde{u}_{h}^{m+1}-\tilde{u}_{h}^{m}}{k}\right)-\frac{g_{i}\left(t_{m+1}\right)-g_{i}\left(t_{m}\right)}{k}\right|=\left\{\begin{array}{l}
O\left(h^{r}+k^{2}\right), \quad \theta=1 / 2 \\
O\left(h^{r}+k\right), \quad \theta>1 / 2 .
\end{array}\right.
$$

Proof. Integrating by parts in (1.1), we have

$$
\left(u_{t}, v\right)+\left(a(x) u_{x}, v_{x}\right)-\left(b(x) u_{x}, v\right)-(F(u, x, t), v)=0, \quad v \in S_{h}^{0} .
$$

Furthermore, using (2.3), (3.1), and (3.7)-(3.8), it follows that

$$
\begin{gathered}
\left(\left(\tilde{u}_{h}\right)_{t}, v\right)-\left(a(x)\left(\tilde{u}_{h}\right)_{x}, v_{x}\right)-\left(b(x)\left(\tilde{u}_{h}\right)_{x}, v\right)-(F(\tilde{u}, x, t), v) \\
=\left(\left(\tilde{u}_{h}\right)_{t}-u_{t}, v\right)-(F(\tilde{u}, x, t)-F(u, x, t), v)
\end{gathered}
$$

Since

$$
\tilde{u}_{h}(t)-u(t)=p_{h}(\hat{u}(t))-\hat{u}(t)+u(\alpha, t)\left(\phi_{0}-v_{0}\right)+u(\beta, t)\left(\phi_{1}-v_{1}\right),
$$

it follows from (2.4), (3.10), that

$$
\left\|\tilde{u}_{h}(t)-u(t)\right\|_{\infty} \leq c h^{r}, \quad\left\|\left(\tilde{u}_{h}\right)_{t}(t)-u_{t}(t)\right\|_{\infty} \leq c h^{r},
$$


from the hypotheses of the theorem, we obtain

$$
\begin{aligned}
& \left\|\tilde{u}_{h}\left(t_{m+\theta}\right)-\left(\theta \tilde{u}_{h}^{m+1}+(1-\theta) \tilde{u}_{h}^{m}\right)\right\|_{\infty}=\left\{\begin{array}{l}
O\left(k^{2}\right), \theta=1 / 2, \\
O(k), \quad \theta>1 / 2,
\end{array}\right. \\
& \left\|\left(\tilde{u}_{h}\right)_{t}\left(m_{t+\theta}\right)-\frac{\tilde{u}_{h}^{m+1}-\tilde{u}_{h}^{m}}{k}\right\|_{\infty}=\left\{\begin{array}{l}
O\left(k^{2}\right), \quad \theta=1 / 2, \\
O(k), \quad \theta>1 / 2,
\end{array}\right.
\end{aligned}
$$

thus, for $0 \leq m \leq M-1$,

$$
\left\|\Gamma^{m+\theta}\left(\tilde{u}_{h}^{m+1}, \tilde{u}_{h}^{m}\right)\right\|_{*}=\left\{\begin{array}{l}
O\left(h^{r}+k^{2}\right), \quad \theta=1 / 2, \\
O\left(h^{r}+k\right), \quad \theta>1 / 2 .
\end{array}\right.
$$

On the other hand

$$
\left|L_{i}\left(\left(\tilde{u}_{h}\right)_{t}(t)\right)-g_{i}^{\prime}(t)\right|=\left|L_{i}\left(\left(\tilde{u}_{h}\right)_{t}(t)-u_{t}(t)\right)\right| \leq c h^{r}, \quad i=0,1,
$$

then, for $0 \leq m \leq M$, we have

$$
\begin{array}{r}
\left|L_{i}\left(\frac{\tilde{u}_{h}^{m+1}-\tilde{u}_{h}^{m}}{k}\right)-\frac{g_{i}\left(t_{m+1}\right)-g_{i}\left(t_{m}\right)}{k}\right| \\
\leq \mid L_{i}\left(\left(\tilde{u}_{h}\right)_{t}\left(t_{m+\theta}\right)-g{ }^{\prime}\left(t_{m+\theta}\right) \mid+O\left(k^{2}\right)\right. \\
=O\left(h^{r}+k^{2}\right), \quad \theta=1 / 2, \quad i=0,1, \\
\left|L_{i}\left(\frac{\tilde{u}_{h}^{m+1}-\tilde{u}_{h}^{m}}{k}\right)-\frac{g_{i}\left(t_{m+1}\right)-g_{i}\left(t_{m}\right)}{k}\right| \\
\leq \mid L_{i}\left(\left(\tilde{u}_{h}\right)_{t}\left(t_{m+\theta}\right)-g_{i}^{\prime}\left(t_{m+\theta}\right) \mid+O(k)\right. \\
=O\left(h^{r}+k\right), \quad \theta>\frac{1}{2}, \quad i=0,1 .
\end{array}
$$

The following theorem ensures the existence of a suitable initial condition that satisfies (2.7), which is required for the $\theta$-method. 
Theorem 3.2. Let $V \in S_{h}$, for $h$ sufficiently small, there exists a unique element $V_{*} \in S_{h}$ such that

$$
\left(a(x)\left(V-V_{*}\right)_{x}, \chi_{x}\right)-\left(b(x)\left(V-V_{*}\right)_{x}, \chi\right)=0, \quad \chi \in S_{h}^{0},
$$

$V_{*}(\alpha)=\int_{\alpha}^{\beta} K_{0}(x) V_{*}(x) d x+g_{0}(\alpha), \quad V_{*}(\beta)=\int_{\alpha}^{\beta} K_{1}(x) V_{*}(x) d x+g_{1}(\alpha)$,

Proof. It suffices to show that if $W$ is such that

$$
\left(a(x) W_{x}, \chi_{x}\right)-\left(b(x) W_{x}, \chi\right)=0, \quad \chi \in S_{h}^{0},
$$

with

$$
W(\alpha)=\int_{\alpha}^{\beta} K_{0}(x) W(x) d x, \quad W(\beta)=\int_{\alpha}^{\beta} K_{1}(x) W(x) d x,
$$

then

$$
W=0 .
$$

(1.6) implies that

$$
|W(\alpha)| \leq \mu\|W\|_{\infty}, \quad|W(\beta)| \leq \mu\|W\|_{\infty}
$$

if we set

$$
\bar{W}=W-W(\alpha) \phi_{0}-W(\beta) \phi_{1},
$$

then $\bar{W} \in S_{h}^{0}$, we have by $(3.15)$

$$
\left(a(x) \bar{W}_{x}, \bar{W}_{x}\right)-\left(b(x) \bar{W}_{x}, \bar{W}\right)=0,
$$

this gives

$$
\bar{W}=0,
$$

thus

$$
W=W(\alpha) \phi_{0}+W(\beta) \phi_{1} .
$$

By (3.6), and for $h$ sufficiently small, we obtain

$$
\|W\|_{\infty} \leq(1+\nu) \max (|W(\alpha)|,|W(\beta)|) \leq(1+\nu) \mu\|W\|_{\infty},
$$

if $\nu$ is chosen so that $(1+\nu) \mu<1$ then $W=0$.

$V_{*}$ can be represented in the form

$$
V_{*}=\bar{V}-V_{*}(\alpha) \phi_{0}-V_{*}(\beta) \phi_{1} .
$$


corollary 3.1. Let $u^{0}$ be the initial condition given in (1.3) and $\tilde{u}_{h}^{0}=$ $\tilde{u}_{h}\left(t_{0}\right)$, for $h$ sufficiently small, if the element $\tilde{u}_{h, *}^{0}$ is given by

$$
\left(a(x)\left(\tilde{u}_{h}^{0}-\tilde{u}_{h, *}^{0}\right)_{x}, \chi_{x}\right)-\left(b(x)\left(\tilde{u}_{h}^{0}-\tilde{u}_{h, *}^{0}\right)_{x}, \chi\right)=0, \quad \chi \in S_{h}^{0},
$$

$\tilde{u}_{h, *}^{0}(\alpha)=\int_{\alpha}^{\beta} K_{0}(x) \tilde{u}_{h, *}^{0}(x) d x+g_{0}(\alpha), \quad \tilde{u}_{h, *}^{0}(\beta)=\int_{\alpha}^{\beta} K_{1}(x) \tilde{u}_{h, *}^{0}(x) d x+g_{1}(\alpha)$,

then

$$
\left\|\tilde{u}_{h, *}^{0}-\tilde{u}_{h}^{0}\right\|_{\infty} \leq C h^{r}
$$

Proof. We use similar techniques as those in [[5], corollary 3.1].

We define in $S_{h}$ the following norm

$$
\|V\|_{S_{h}}^{2}=\left(a \bar{V}_{x}, \bar{V}_{x}\right)-\left(b \bar{V}_{x}, \bar{V}\right)+\|V\|_{\infty}^{2}
$$

for $\left(V^{0}, \ldots, V^{M}\right) \in S_{h}^{M+1}$

$$
\left\|\left(V^{0}, \ldots, V^{M}\right)\right\|_{S_{h}^{M+1}}=\max _{0 \leq m \leq M}\left\|V^{m}\right\|_{\infty},
$$

and

$$
\begin{aligned}
& \left\|\left(V,\left(\Gamma^{m+\theta}\right)_{m=1}^{M},\left(a_{m}\right)_{m=1}^{M},\left(b_{m}\right)_{m=1}^{M}\right)\right\|_{Y_{h k}}^{2} \\
& =\|V\|_{S_{h}}^{2}+\sum_{m=1}^{M} k\left\|\Gamma^{m+\theta}\right\|_{*}^{2}+\sum_{m=1}^{M} k\left|a_{m}\right|^{2}+\sum_{m=1}^{M} k\left|b_{m}\right|^{2}
\end{aligned}
$$

corollary 3.2. Under the hypotheses of theorem 3.1, if $\tilde{u}_{h}(x, t)$ is the function defined by (3.8) and the initial condition is chosen such that $U^{0}=\tilde{u}_{h, *}$, where $\tilde{u}_{h, *} \in S_{h}$ is defined in corollary (3.1), and $\theta \geq 1 / 2$, then

$$
\left\|\Phi_{h k}\left(\tilde{u}_{h}^{0}, \ldots, \tilde{u}_{h}^{N}\right)\right\|_{Y_{h k}}=\left\{\begin{array}{l}
O\left(h^{r}+k^{2}\right), \quad \theta=1 / 2, \\
O\left(h^{r}+k\right), \quad \theta>1 / 2 .
\end{array}\right.
$$




\section{Existence and convergence}

We first, show a stability result of the $\theta$-method.

Theorem 4.1. Let $R$ be a fixed real positif and assume that $u \in C^{r, 3}([\alpha, \beta] \times[0, T])$, for $h$ and $k$ sufficiently small, $\left(V^{0}, \ldots, V^{M}\right)$ and $\left(W^{0}, \ldots, W^{M}\right) \in B\left(\left(\tilde{u}_{h}^{0}, \ldots, \tilde{u}_{h}^{M}\right), R\right)$ there exists a positive constant $S$ such that

$$
\begin{aligned}
& \left\|\left(V^{0}, \ldots, V^{M}\right)-\left(W^{0}, \ldots, W^{M}\right)\right\|_{X_{h k}} \\
& \leq S\left\|\Phi_{h k}\left(V^{0}, \ldots, V^{M}\right)-\Phi_{h k}\left(W^{0}, \ldots, W^{M}\right)\right\|_{Y_{h k}} .
\end{aligned}
$$

where $B\left(\left(\tilde{u}_{h}^{0}, \ldots, \tilde{u}_{h}^{M}\right), R\right)$ is the ball of center $\left(\tilde{u}_{h}^{0}, \ldots, \tilde{u}_{h}^{M}\right)$ and radius $R$.

Proof. Noting that for $V \in S_{h}, \bar{V} \in S_{h}^{\circ}$ is given by

$$
\bar{V}=V-V(\alpha) \phi_{0}-V(\beta) \phi_{1} .
$$

For $\left(V^{0}, \ldots, V^{M}\right)$ and $\left(W^{0}, \ldots, W^{M}\right) \in B\left(\left(\tilde{u}_{h}^{0}, \ldots, \tilde{u}_{h}^{M}\right), R\right)$, we set $R^{m}=V^{m}-W^{m}, 0 \leq m \leq M$.

$$
\begin{aligned}
\Gamma^{m+\theta}\left(V^{m}, V^{m+1}\right)\left(\frac{\bar{R}^{m+1}-\bar{R}^{m}}{k}\right)- & \Gamma^{m+\theta}\left(W^{m}, W^{m+1}\right)\left(\frac{\bar{R}^{m+1}-\bar{R}^{m}}{k}\right) \\
=\left(\frac{R^{m+1}-R^{m}}{k}, \frac{\bar{R}^{m+1}-\bar{R}^{m}}{k}\right)+ & \left(a R_{x}^{m+\theta}, \frac{\bar{R}_{x}^{m+1}-\bar{R}_{x}^{m}}{k}\right) \\
& -\left(b R_{x}^{m+\theta}, \frac{\bar{R}^{m+1}-\bar{R}^{m}}{k}\right)
\end{aligned}
$$

$$
-\left(F\left(V^{m+\theta}, t_{m+\theta}\right)-F\left(W^{m+\theta}, t_{m+\theta}\right), \frac{\bar{R}^{m+1}-\bar{R}^{m}}{k}\right),
$$

for $0 \leq m \leq M-1$. Then

$$
\begin{gathered}
\left\|\Gamma^{m+\theta}\left(V^{m}, V^{m+1}\right)\left(\frac{\bar{R}^{m+1}-\bar{R}^{m}}{k}\right)-\Gamma^{m+\theta}\left(W^{m+1}\right)\left(\frac{\bar{R}^{m+1}-\bar{R}^{m}}{k}\right)\right\|^{2} \\
\leq\left\|\Gamma^{m+\theta}\left(V^{m}, V^{m+1}\right)-\Gamma^{m+\theta}\left(W^{m}, W^{m+1}\right)\right\|_{*}^{2}\left\|\frac{\bar{R}^{m+1}-\bar{R}^{m}}{k}\right\|^{2},
\end{gathered}
$$


taking into account (3.1) we get

$$
\begin{aligned}
& \left(a R_{x}^{m+\theta}, \frac{\bar{R}_{x}^{m+1}-\bar{R}_{x}^{m}}{k}\right)-\left(b R_{x}^{m+\theta}, \frac{\bar{R}^{m+1}-\bar{R}^{m}}{k}\right)
\end{aligned}
$$

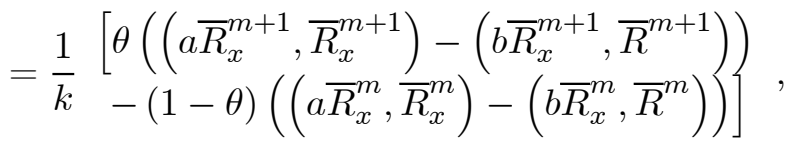

hence

$$
\begin{gathered}
\frac{1}{k} \quad\left[\theta\left(\left(a \bar{R}_{x}^{m+1}, \bar{R}_{x}^{m+1}\right)-\left(b \bar{R}_{x}^{m+1}, \bar{R}^{m+1}\right)\right)\right\} \\
\left.\leq \| \Gamma^{M+\theta}\left(V^{m}, V^{m+1}\right)-\Gamma^{m+\theta}\left(\left(a \bar{R}_{x}^{m}, \bar{R}_{x}^{m}\right)-\left(b \bar{R}_{x}^{m}, \bar{R}^{m}\right)\right)\right] \\
+\left\|F\left(V^{m+\theta}, t_{m+\theta}\right)-F\left(W^{m+\theta}, t_{m+\theta}\right)\right\|_{*}^{2} \\
+\frac{1}{2}\left\|\frac{\bar{R}^{m+1}-\bar{R}^{m}}{k}\right\|^{2}-\left(\frac{\bar{R}^{m+1}-\bar{R}^{m}}{k}, \frac{R^{m+1}-R^{m}}{k}\right),
\end{gathered}
$$

Moreover

$$
\begin{aligned}
\left(\frac{\bar{R}^{m+1}-\bar{R}^{m}}{k}, \frac{R^{m+1}-R^{m}}{k}\right) & \\
=\left\|\frac{R^{m+1}-R^{m}}{k}\right\|^{2} & -\left(\frac{R^{m+1}-R^{m}}{k}, \frac{R^{m+1}(\alpha)-R^{m}(\alpha)}{k} \emptyset_{0}\right) \\
& -\left(\frac{R^{m+1}-R^{m}}{k}, \frac{R^{m+1}(\beta)-R^{m}(\beta)}{k} \emptyset_{1}\right),
\end{aligned}
$$

and

$$
\begin{aligned}
\left\|\frac{\bar{R}^{m+1}-\bar{R}^{m}}{k}\right\|^{2} & =\left\|\frac{R^{m+1}-R^{m}}{k}\right\|^{2} \\
- & 2\left(\frac{R^{m+1}-R^{m}}{k}, \frac{R^{m+1}(\alpha)-R^{m}(\alpha)}{k} \emptyset_{0}\right) \\
& -2\left(\frac{R^{m+1}-R^{m}}{k}, \frac{R^{m+1}(\beta)-R^{m}(\beta)}{k} \emptyset_{1}\right)
\end{aligned}
$$




$$
\begin{aligned}
& +\left|\frac{R^{m+1}(\alpha)-R^{m}(\alpha)}{k}\right|^{2}\left\|\emptyset_{0}\right\|^{2}+\left|\frac{R^{m+1}(\beta)-R^{m}(\beta)}{k}\right|^{2}\left\|\emptyset_{1}\right\|^{2} \\
& +2\left(\frac{R^{m+1}(\alpha)-R^{m}(\alpha)}{k} \emptyset_{0}, \frac{R^{m+1}(\beta)-R^{m}(\beta)}{k} \emptyset_{1}\right) .
\end{aligned}
$$

It follows by replacing all these inequalities in (4.4) that

$$
\begin{gathered}
\frac{1}{\bar{k}} \quad\left[\theta\left(\left(a \bar{R}_{x}^{m+1}, \bar{R}_{x}^{m+1}\right)-\left(b R_{x}^{m+1}, R_{x}^{m+1}\right)\right)\right. \\
\leq\left\|\Gamma^{m+\theta}\left(V^{m}, V^{m+1}\right)-\Gamma^{m+\theta}\left(W^{m}, W^{m+1}\right)\right\|_{*}^{2} \\
\left.\left.+\| F\left(V^{m+\theta}, t_{m+\theta}^{m}\right)-F\left(\bar{R}_{x}^{m}, \bar{R}^{m}\right)\right)\right] \\
-\frac{1}{2}\left\|\frac{R^{m+1}-R^{m}}{k}\right\|^{2}+\frac{1}{2}\left|\frac{R^{m+1}(\alpha)-R^{m}(\alpha)}{k}\right|^{2}\left\|\emptyset_{0}\right\|^{2} \\
+\frac{1}{2}\left|\frac{R^{m+1}(\beta)-R^{m}(\beta)}{k}\right|^{2}\left\|\emptyset_{1}\right\|^{2} \\
+\left|\frac{R^{m+1}(\alpha)-R^{m}(\alpha)}{k}\right|\left|\frac{R^{m+1}(\beta)-R^{m}(\beta)}{k}\right|\left(\left|\emptyset_{0}\right|,\left|\emptyset_{1}\right|\right),
\end{gathered}
$$

for $h$ sufficiently small, the last three terms of (4.6) are bounded by

$$
\begin{array}{r}
\frac{1}{4}\left|\frac{R^{m+1}(\alpha)-R^{m}(\alpha)}{k}\right|^{2}\left(1+\left\|\emptyset_{0}\right\|^{2}-\left\|\emptyset_{1}\right\|^{2}+\nu\right) \\
+\frac{1}{4}\left|\frac{R^{m+1}(\beta)-R^{m}(\beta)}{k}\right|^{2}\left(1+\left\|\emptyset_{1}\right\|^{2}-\left\|\emptyset_{0}\right\|^{2}+\nu\right),
\end{array}
$$

combining (1.6), the Cauchy-Schwartz inequality, and the fact that $(a+b)^{2} \leq$ $(1+\delta) a^{2}+\left(1+\frac{1}{\delta}\right) b^{2}$, for any real numbers $a, b$ and for any $\delta>0$ we get

$$
\begin{aligned}
& \left|\frac{R^{m+1}(\alpha)-R^{m}(\alpha)}{k}\right|^{2} \leq\left(1+\frac{\delta}{4}\right) \mu^{2}\left\|\frac{R^{m+1}-R^{m}}{k}\right\|^{2}+\left(1+\frac{4}{\delta}\right)\left|L_{0}\left(\frac{R^{m+1}-R^{m}}{k}\right)\right|^{2} \\
& \left|\frac{R^{m+1}(\beta)-R^{m}(\beta)}{k}\right|^{2} \leq\left(1+\frac{\delta}{4}\right) \mu^{2}\left\|\frac{R^{m+1}-R^{m}}{k}\right\|^{2}+\left(1+\frac{4}{\delta}\right)\left|L_{1}\left(\frac{R^{m+1}-R^{m}}{k}\right)\right|^{2},
\end{aligned}
$$


If we substitute (4.7)-(4.8) into (4.6), we obtain

$$
\begin{gathered}
\theta\left(\left(a \bar{R}_{x}^{m+1}, \bar{R}_{x}^{m+1}\right)-\left(b \bar{R}_{x}^{m+1}, \bar{R}^{m+1}\right)\right)-(1-\theta)\left(\left(a \bar{R}_{x}^{m}, \bar{R}_{x}^{m}\right)-\left(b \bar{R}_{x}^{m}, \bar{R}^{m}\right)\right) \\
\leq k\left\|\Gamma^{m+\theta}\left(V^{m}, V^{m+1}\right)-\Gamma^{m+\theta}\left(W^{m}, W^{m+1}\right)\right\|_{*}^{2} \\
+k\left\|F\left(V^{m+\theta}, t_{m+\theta}\right)-F\left(W^{m+\theta}, t_{m+\theta}\right)\right\|^{2} \\
+k\left(\frac{1+\left\|\phi_{0}\right\|^{2}-\left\|\phi_{1}\right\|^{2}+\nu}{4}\right)\left(1+\frac{4}{\delta}\right)\left|L_{0}\left(\frac{R^{m+1}-R^{m}}{k}\right)\right|^{2} \\
+k\left(\frac{1+\left\|\phi_{1}\right\|^{2}-\left\|\phi_{0}\right\|^{2}+\nu}{4}\right)\left(1+\frac{4}{\delta}\right)\left|L_{1}\left(\frac{R^{m+1}-R^{m}}{k}\right)\right|^{2} \\
(4.9) \quad-\frac{k}{2}\left[1-(1+\nu) \mu^{2}(\beta-\alpha)\left(1+\frac{\delta}{4}\right)\right]\left\|\frac{R^{m+1}-R^{m}}{k}\right\|^{2},
\end{gathered}
$$

$0 \leq m \leq M-1$. If $\nu$ and $\delta$ are chosen such that $(1+\nu) \mu^{2}\left(1+\frac{\delta}{4}\right)<1$ and using the fact that the function $F$ is Lipschitz continuous (4.8) takes the form

$$
\begin{gathered}
\frac{\theta}{1-\theta}\left(\left(a \bar{R}_{x}^{m+1}, \bar{R}_{x}^{m+1}\right)-\left(b \bar{R}_{x}^{m+1}, \bar{R}^{m+1}\right)\right)-\left(\left(a \bar{R}_{x}^{m}, \bar{R}_{x}^{m}\right)-\left(b \bar{R}_{x}^{m}, \bar{R}^{m}\right)\right) \\
\leq \frac{k}{1-\theta}\left\|\Gamma^{m+\theta}\left(V^{m}, V^{m+1}\right)-\Gamma^{m+\theta}\left(W^{m}, W^{m+1}\right)\right\|_{*}^{2} \\
+\frac{C k}{1-\theta}\left(\left\|R^{m+1}\right\|^{2}+\left\|R^{m}\right\|^{2}\right) \\
(4.10)+\frac{C k}{1-\theta}\left(\left|L_{0}\left(\frac{R^{m+1}-R^{m}}{k}\right)\right|^{2}+\left|L_{1}\left(\frac{R^{m+1}-R^{m}}{k}\right)\right|^{2}\right),
\end{gathered}
$$

$0 \leq m \leq M-1$, summing on $m$ we have

$$
\begin{aligned}
& \left(a \bar{R}_{x}^{m}, \bar{R}_{x}^{m}\right)-\left(b \bar{R}_{x}^{m}, \bar{R}^{m}\right)-\left(\frac{1-\theta}{\theta}\right)^{m}\left(\left(a \bar{R}_{x}^{0}, \bar{R}^{0}\right)-\left(b \bar{R}_{x}^{0}, \bar{R}^{0}\right)\right) \\
\leq & C\left(\sum_{n=1}^{m} \frac{(1-\theta)^{m-n}}{\theta^{m-n+1}} k\left\|\Gamma^{n-1+\theta}\left(V^{m}, V^{m-1}\right)-\Gamma^{n-1+\theta}\left(W^{m}, W^{m-1}\right)\right\|_{*}^{2}\right. \\
+ & \sum_{n=1}^{m} \frac{(1-\theta)^{m-n}}{\theta^{m-n+1}} k\left[\left|L_{0}\left(\frac{R^{n}-R^{n-1}}{k}\right)\right|^{2}+\left|L_{1}\left(\frac{R^{n}-R^{n-1}}{k}\right)\right|^{2}\right]
\end{aligned}
$$


(4.11) $\left.+\sum_{n=0}^{m} \frac{(1-\theta)^{m-n-1}}{\theta^{m-n+1}} k\left\|R^{n}\right\|^{2}+\frac{(1-\theta)^{m-1}}{\theta^{m}} k\left\|R^{0}\right\|^{2}+\frac{k}{\theta}\left\|R^{m}\right\|^{2}\right)$

$1 \leq m \leq M$.

Since $1 / 2 \leq \theta \leq 1$ then $\frac{1-\theta}{\theta} \leq 1$.

On the other hand, using Sobolev's inequality we get

$$
\left\|R^{m}\right\|_{\infty} \leq C\left(\left\|\bar{R}_{x}^{m}\right\|+\left\|\bar{R}^{m}\right\|+\left|L_{0}\left(R^{m}\right)\right|+\left|L_{1}\left(R^{m}\right)\right|\right),
$$

and

$$
\left|L_{i}\left(R^{m}\right)\right|^{2} \leq 2 T \sum_{j=1}^{m} k\left|L_{i}\left(\frac{R^{j}-R^{j-1}}{k}\right)\right|^{2}+\left|L_{i}\left(R^{0}\right)\right|^{2}, \quad i=0,1,
$$

from (1.4)

$a_{0}\left\|\bar{R}_{x}^{m}\right\|^{2}+1 / 2 b_{0}\left\|\bar{R}^{m}\right\|^{2} \leq\left(a \bar{R}_{x}^{m}, \bar{R}_{x}^{m}\right)-\left(b \bar{R}_{x}^{m}, \bar{R}^{m}\right) \leq a_{1}\left\|\bar{R}_{x}^{m}\right\|^{2}+1 / 2 b_{1}\left\|\bar{R}^{m}\right\|^{2}$, (4.14)

thus

$$
\begin{gathered}
\left\|R^{m}\right\|_{\infty}^{2} \leq C\left(\left(a \bar{R}_{x}^{0}, \bar{R}_{x}^{0}\right)-\left(b \bar{R}_{x}^{0}, \bar{R}^{0}\right)+\left|L_{0}\left(R^{0}\right)\right|^{2}+\left|L_{1}\left(R^{0}\right)\right|^{2}\right. \\
+\sum_{n=1}^{m} k\left\|\Gamma^{n-1+\theta}\left(V^{n}, V^{n-1}\right)-\Gamma^{n-\theta}\left(W^{n}, W^{n-1}\right)\right\|_{*}^{2}+k\left\|R^{0}\right\|^{2} \\
\left.+\sum_{n=1}^{m} k\left[\left|L_{0}\left(\frac{R^{n}-R^{n-1}}{k}\right)\right|^{2}+\left|L_{1}\left(\frac{R^{n}-R^{n-1}}{k}\right)\right|^{2}\right]+\sum_{m=1}^{n} k\left\|R^{n}\right\|_{\infty}^{2}\right),
\end{gathered}
$$

to conclude, we use Gronwal's inequality (discrete form), which gives, for $k$ sufficiently small,

$$
\begin{aligned}
\left\|R^{m}\right\|_{\infty}^{2} & \leq C\left(\left\|R^{0}\right\|_{S_{h}}^{2}+\sum_{n=1}^{m} k\left\|\Gamma^{n-\theta}\left(V^{n}, V^{n-1}\right)-\Gamma^{n-\theta}\left(W^{n}, W^{n-1}\right)\right\|_{*}^{2}\right. \\
& \left.+\sum_{n=1}^{m}\left[\left|L_{0}\left(\frac{R^{n}-R^{n-1}}{k}\right)\right|^{2}+\left|L_{1}\left(\frac{R^{n}-R^{n-1}}{k}\right)\right|^{2}\right]\right)
\end{aligned}
$$

for $0 \leq m \leq M$, thus

$$
\left\|\left(V^{0}, \ldots, V^{M}\right)-\left(W^{0}, \ldots, W^{M}\right)\right\|_{X_{h k}}
$$

$\leq S\left\|\Phi_{h k}\left(V^{0}, \ldots, V^{M}\right)-\Phi_{h k}\left(W^{0}, \ldots, W^{M}\right)\right\|_{Y_{h k}}$, which completes the proof.

Analogous to theorem 5.1 of [5], the following result holds: 
Theorem 4.2. Let be $\left(\tilde{u}_{h}^{0}, \ldots, \tilde{u}_{h}^{M}\right)$ defined as in (3.9) and $\Phi_{h k}$ the operator defined as in (2.5) with $U^{0}=\tilde{u}_{h, *}^{0}$ if $R$ is a fixed positif real, for $h$ and $k$ sufficiently small, (2.8) possesses a unique solution $\left(U^{0}, \ldots, U^{M}\right) \in$ $B\left(\left(\tilde{u}_{h}^{0}, \ldots, \tilde{u}_{h}^{M}\right), R\right)$ that satisfies

$$
\left\|\left(U^{0}, \ldots, U^{M}\right)-\left(\tilde{u}_{h}^{0}, \ldots, \tilde{u}_{h}^{M}\right)\right\|_{Y_{h k}}=\left\{\begin{array}{l}
O\left(h^{r}+k^{2}\right), \quad \theta=1 / 2, \\
O\left(h^{r}+k\right), \quad \theta>1 / 2 .
\end{array}\right.
$$

The following corollary is a consequence of the above theorem.

corollary 4.1. For $\theta \geq 1 / 2$, under the hypotheses of theorem 4.2, if

$\left(U^{0}, \ldots, U^{M}\right)$ is the solution of (2.8), and consider

$$
u^{m}(x)=u\left(x, t_{m}\right), \quad 0 \leq m \leq M,
$$

where $u$ is the solution of (1.1-1.3), then

$$
\left\|U^{m}-u^{m}\right\|_{\infty}=\left\{\begin{array}{l}
O\left(h^{r}+k^{2}\right), \quad \theta=1 / 2, \\
O\left(h^{r}+k\right), \quad \theta>1 / 2, \quad 0 \leq m \leq M .
\end{array}\right.
$$

\section{Numerical results}

To observe the behavior of the $\theta$ - method, it was tested on the following problem [1].

$$
\begin{gathered}
u_{t}-u_{x x}=-e^{-t}\left\{x(x-1)+\frac{\delta}{6(1+\delta)}+2\right\}, \quad x \in(0,1), \quad 0<t \leq T, \\
u(0, t)=u(1, t)=-\delta \int_{0}^{1} u(x, t) d x, \quad t>0, \\
u(x, 0)=x(x-1)+\frac{\delta}{6(1+\delta)}, \quad x \in[0,1] .
\end{gathered}
$$

Where $|\delta|<1$ and the exact solution, $u=u(x, t)$, is given by

$$
u(x, t)=e^{-t}\left(x(x-1)+\frac{\delta}{6(1+\delta)}\right) .
$$

Below are tables, representing sample calculations using three values for the parameter $\theta$ of the described methods. The first and second column in the table represents the mesh parameter $M$ and the time steps $N$. The third column shows the error measured in maximum norm on the $M \times N$ mesh, covering $[0,1] \times[0, T]$ and the last column shows the CPU-time used, in seconds, for the computation. 
Table 5.1: Crank-Nicholson method, $\theta=\frac{1}{2}$

\begin{tabular}{|c|c|c|c|}
\hline $\mathrm{M}$ & $\mathrm{N}$ & error & CPU-time \\
\hline 2 & 2 & $4.04 \mathrm{e}-04$ & $8.61 \mathrm{e}-01$ \\
4 & 4 & $2.19 \mathrm{e}-04$ & $9.1 \mathrm{e}-01$ \\
8 & 8 & $7.07 \mathrm{e}-05$ & $9.8 \mathrm{e} 00$ \\
16 & 16 & $2.37 \mathrm{e}-05$ & $5.22 \mathrm{e} 01$ \\
32 & 32 & $3.55 \mathrm{e}-06$ & $6.0 \mathrm{e} 02$ \\
64 & 64 & $1.87 \mathrm{e}-06$ & $1.2 \mathrm{e} 03$ \\
128 & 128 & $6.35 \mathrm{e}-07$ & $3.9 \mathrm{e} 03$ \\
\hline
\end{tabular}

Table 5.2: $\theta=0$

\begin{tabular}{|c|c|c|c|}
\hline $\mathrm{M}$ & $\mathrm{N}$ & error & CPU-time \\
\hline 2 & 2 & $7.21 \mathrm{e}-04$ & $9.21 \mathrm{e}-01$ \\
4 & 4 & $9.8 \mathrm{e}-04$ & $1.1 \mathrm{e} 00$ \\
8 & 8 & $8.04 \mathrm{e}-05$ & $1.03 \mathrm{e} 01$ \\
16 & 16 & $5.87 \mathrm{e}-05$ & $6.34 \mathrm{e} 01$ \\
32 & 32 & $2.45 \mathrm{e}-05$ & $7.21 \mathrm{e} 02$ \\
64 & 64 & $7.25 \mathrm{e}-06$ & $2.4 \mathrm{e} 03$ \\
128 & 128 & $3.23 \mathrm{e}-06$ & $5.8 \mathrm{e} 03$ \\
\hline
\end{tabular}


Table 5.3: $\theta=1$

\begin{tabular}{|c|c|c|c|}
\hline $\mathrm{M}$ & $\mathrm{N}$ & error & CPU-time \\
\hline 2 & 2 & $8.4 \mathrm{e}-04$ & $1.6 \mathrm{e} 00$ \\
4 & 4 & $5.14 \mathrm{e}-04$ & $2.11 \mathrm{e} 00$ \\
8 & 8 & $3.7 \mathrm{e}-04$ & $13.98 \mathrm{e} 00$ \\
16 & 16 & $8.27 \mathrm{e}-05$ & $5.22 \mathrm{e} 01$ \\
32 & 32 & $3.5 \mathrm{e}-05$ & $8.59 \mathrm{e} 02$ \\
64 & 64 & $8.67 \mathrm{e}-06$ & $2.52 \mathrm{e} 03$ \\
128 & 128 & $4.86 \mathrm{e}-06$ & $7.75 \mathrm{e} 03$ \\
\hline
\end{tabular}

Comparing the above three tables, we find that the Crank-Nicholson method is, unsurprisingly, more efficient than the others.

\section{References}

[1] A. Boutayeb, A. Chetouani, Global extrapolations of numerical methods for a parabolic problem with nonlocal boundary conditions, International journal of computer mathematics 80, No. 6, pp. 789-797, (2003).

[2] W. A. DAY, Extension of a property of the heat equation to linear thermoelasticity and other theories, Quart. Appl. Math. 40, pp. 319330, (1982).

[3] W. A. DAY, A decreasing property of solutions of parabolic equations with apllications to thermoelasticity, Quart. Appl. Math. 40, pp. 468475, (1983).

[4] G. Ekolin, Finite difference methods for a nonlocal boundary value problem for the heat equation, BIT 31, pp. 245-261, (1991).

[5] G. Fairweather, J.C. López-Marcos, Galerkin methods for a semilinear parabolic problem with nonlocal boundary conditions, Advances in Computational mathematics 6, pp. 243-262, (1996). 
[6] A. Friedman, Monotonic decay of solutions of parabolic equations with nonlocal boundary conditions, Quart. Appl. Math. 44, pp. 401407, (1986).

[7] A. Kawohl, Remarks on a paper by W. A. Day on a maximun principle under nonlocal boundary conditions, Quart. Appl. Math. 44, pp. 751$752,(1987)$.

[8] J. C. López-Marcos, J. M.Sanz-Serna, Stability and convergence in numerical analysis III: Linear investigation of nonlinear stability, IMAJ. Numer. Anal. 8, pp. 71-84, (1988)

[9] M. F. Wheeler, An optimal $L_{\infty}$ error estimate for Galerkin approximation to solutions of two boudary value problems, SIAMJ. Numer. Anal 10, pp. 914-917, (1973).

[10] J. C. López-Marcos, J. M.Sanz-Serna, A definition of stability for nonlinear problems, in: Numerical Treatment of Differential Equations, ed. K. Strehmel, Teubner Texte zur Mathematic (Teubner, Leipzig, pp. 216-226, (1988).

[11] G. Fairweather, J.C. López-Marcos, A.Boutayeb, Orthogonal Spline Collocation for a Quasilinear parabolic problem with nonlocal boundary conditions, Preprint.

\author{
Abdesslam Boutayeb \\ Département de Mathématique \\ Faculté des Sciences \\ Université Mohammed Premier \\ 60000 Oujda \\ Maroc \\ e-mail : boutayeb@sciences.univ-oujda.ac.ma \\ and
}

\author{
Abdelaziz Chetouani \\ Département de Mathématique \\ Faculté des Sciences \\ Université Mohammed Premier \\ 60000 Oujda \\ Maroc \\ e-mail : a-chetouani@sciences.univ-oujda.ac.ma
}

